\title{
Who plays in the Hoyle band?
}

\author{
Tzany Kokalova ${ }^{1, a}$ \\ ${ }^{1}$ School of Physics and Astronomy, University of Birmingham, Edgbaston, Birmingham, B15 2TT, United \\ Kingdom
}

\begin{abstract}
The Hoyle state in ${ }^{12} \mathrm{C}$ was discovered over 50 years ago in 1957 and strongly influences the rate of the triple-alpha process in stars. Recently, significant experimental and theoretical effort has been invested to understand, in detail, its unorthodox structure. In particular, identification of excitations of the Hoyle state has been a key goal. This paper addresses the current progress with an emphasis on possible members of the Hoyle band and the structure implications.
\end{abstract}

\section{Introduction}

The fusion of three $\alpha$-particles into ${ }^{12} \mathrm{C}$ is able to proceed at a rate sufficient to make carbon-based life possible [1], only due to the serendipitous occurrence of two resonances. The first is the ground-state of ${ }^{8} \mathrm{Be}$ [2], $91.8 \mathrm{keV}$ above the threshold for $\alpha$ decay. The second is the Hoyle state [3], $287 \mathrm{keV}$ above the $\alpha$ decay threshold. Both resonances have widths of the order of $\mathrm{eV}$ and the corresponding lifetimes $\left(\sim 10^{-17} \mathrm{~s}\right)$ are sufficient to lead to an equilibrium quantity of ${ }^{8} \mathrm{Be}\left(\alpha+\alpha \rightleftharpoons{ }^{8} \mathrm{Be}\right)$ and ${ }^{12} \mathrm{C}^{*}$ $\left(\alpha+{ }^{8} \mathrm{Be} \rightleftharpoons{ }^{12} \mathrm{C}^{*}\right)$ in stellar nucleosynthesis environments.

Not only is the presence of the Hoyle state close to the $\alpha$-decay threshold key, but its properties are of paramount importance. For example, it is not sufficient to produce ${ }^{12} \mathrm{C}$ in an excited state, but a significant route to the ground state is required. In the case of the Hoyle state, a $0.04 \%$ radiative branching ratio to bound states has been determined [4]. The dominant decay process undergone by the Hoyle state, as outlined above, is via the ground-state of ${ }^{8} \mathrm{Be}$, with a limit on the direct disintegration into three $\alpha$ particles having recently been reported as $<5 \times 10^{-3}$ [5].

Over the past few years, intense activity has been focused on attempting to measure excitations of the Hoyle state as a means to measure not only the moment-of-inertia (assuming rotationaly behaviour) of the Hoyle band and its underlying structure with precision, but also to obtain accurate energies for the location of the excitations, to enable their influence on the stellar carbon-production to be modelled. These efforts also capture the speculation involving nuclear condensates [6], comprising an $\alpha$-Bose gas in which each $\alpha$ particle shares a common wave function and for which the Hoyle state is considered a prime candidate [6].

This paper considers recent experimental and theoretical results relating to the Hoyle state and its excitations.

\footnotetext{
a e-mail: t.kokalova@bham.ac.uk
} 


\section{Clustering and the Hoyle state}

Clustering in nuclei is well established $[7,8]$ with the usual picture of homogeneously mixed protons and neutrons being replaced by diverse structures comprising sub-clusters of nucleons arranged together. Examples include the symmetric ${ }^{12} \mathrm{C}-{ }^{12} \mathrm{C}$ cluster resonance in ${ }^{24} \mathrm{Mg}$ [8] and the well developed ${ }^{16} \mathrm{O}-{ }^{4} \mathrm{He}$ structure of ${ }^{20} \mathrm{Ne}$ [9]. Alpha particles are by far the most ubiquitous sub-units within cluster states, arising from their strongly bound nature; the particle threshold at $19.81 \mathrm{MeV}$ makes the $\alpha$ particle the most robust composite nucleus in the entire Segrè chart. This is evidenced by the particularly high binding energies for $\alpha$-conjugate nuclei $\left({ }^{8} \mathrm{Be},{ }^{12} \mathrm{C},{ }^{16} \mathrm{O}\right.$ etc.). Ikeda [10], associated the different clustering opportunities for specific nuclei with the corresponding particle decay thresholds - the energy needed to dissolve the nucleus into a particular set of cluster sub-units - encompassed in the Ikeda diagram shown in Fig. 1.

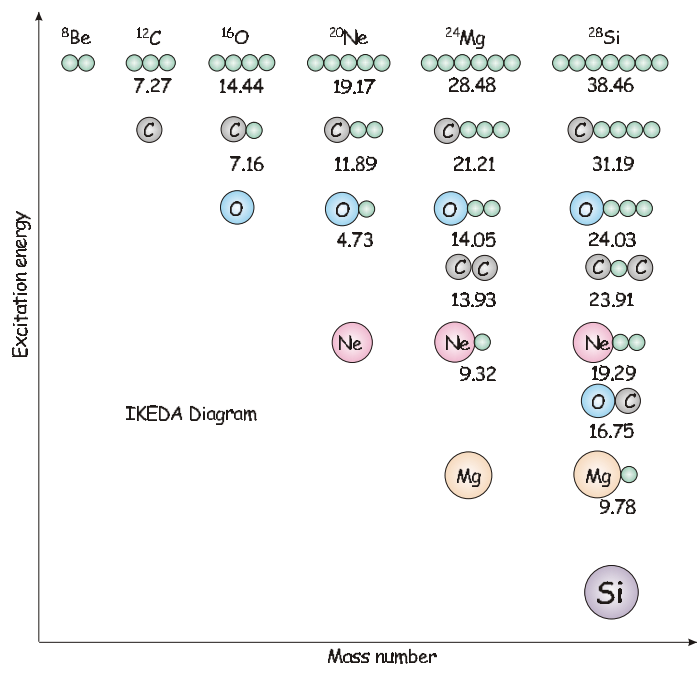

Figure 1. The Ikeda diagram [10], as designed by the current author and appearing in Ref. [7]. The decay thresholds are given in $\mathrm{MeV}$ below particular arrangements of clusters. Alpha particles are green and heavier $\alpha$-conjugate nuclei are colour-coded and labelled by element.

As can be seen from Fig. 1, the Hoyle state $\left(E_{x}=7.654 \mathrm{MeV}\right)$ lies just above the three $\alpha$ threshold at 7.27 MeV. For heavy nuclei, the multi- $\alpha$-cluster thresholds lie at very high energies, e.g. at 14.44 $\mathrm{MeV}$ in ${ }^{16} \mathrm{O}$, making Hoyle-state analogues increasingly difficult to study due to their being embedded in a high density of (typically broad) resonances. Returning to the Hoyle state itself, evidence for a cluster structure comes largely from $\alpha$ decay widths and branching ratios. Beryllium- 8 is perhaps the most trivial clustered nucleus, comprised of two $\alpha$-particles, and the Hoyle state preferentially decays to this structure. This is indicative of a shared structure for the Hoyle state and ${ }^{8} \mathrm{Be}$ daughter. Indeed, the Hoyle state has a reduced $\alpha$-decay width of approximately $100 \%$ when compared to the first Wigner sum rule (or single-particle limit) $[11,12]$. The clustered nature of the ${ }^{8} \mathrm{Be}$ ground-state has been within reach of $a b$ initio calculations starting with a nucleon-nucleon interaction and using the Green's Function Monte Carlo (GFMC) approach. Wiringa et al. [13] demonstrated that clustering comprising two- $\alpha$ particles emerges naturally in such calculations. The density distribution is shown in Fig. 2. When extending the "clustering" picture to neutron-rich systems, an analogy with molecules can be made. Neutrons are shared between the cluster-unit cores and provide additional binding; akin to covalent bonding in between atoms. The neutrons can lie on- or off-axis leading to $\sigma$ and $\pi$ bonds respectively. Two particularly good examples of this are ${ }^{10} \mathrm{Be}$ (see Fig. 3) [14] and ${ }^{21} \mathrm{Ne}$ [15]. This is especially relevant when considering how relatives of the Hoyle state would appear in heavier carbon isotopes such as ${ }^{13} \mathrm{C}$ and ${ }^{14} \mathrm{C}$. 


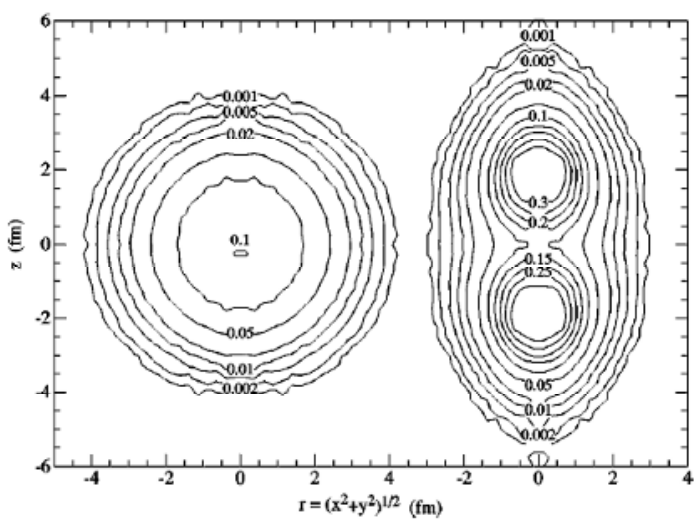

Figure 2. Density distributions for the ${ }^{8} \mathrm{Be}$ ground state arising from GFMC calculations (reproduced from Ref. [13]). Contours of constant density are shown for the intrinsic (left) and laboratory (right) frames using cylindrical polar coordinates.

Additionally, over the last decade, interest has increased in a possible new form of nuclear matter, namely, Bose-gas-like states or condensates. The Hoyle state is considered as the best case for such a state of matter and a testing ground for the models $[6,16,17]$. In this picture, $\alpha$ particles all share the same $s$-orbit and the structure of the state is extended. Since $\alpha$ particles are correlated in such a system the decay is affected, and, due to the increased radius, the Coulomb barrier reduced [18]. The hypothesis is that this can give rise to significant differences in carbon-12 production in stars.

In both the clustered and condensed scenarios, excitations are allowed and in the latter take the form of exciting one of the $\alpha$ particles to a $d$-orbit $[19,20]$. Therefore, by observing excitations of the Hoyle state, much information on its underlying structure can be garnered.

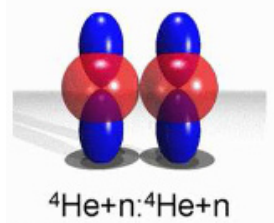

Bonding $\pi$

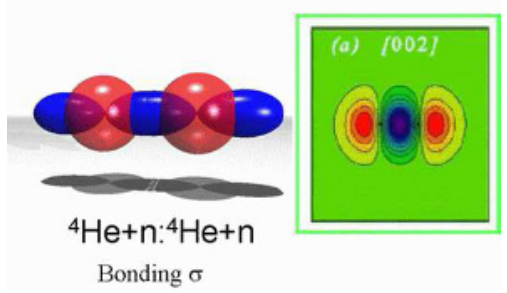

Figure 3. Graphical representation of the 'nuclear molecule', ${ }^{10} \mathrm{Be}$. In the left-hand column, the red spheres are $\alpha$ particles and the blue lobes are neutron-orbitals: (top row) off-axis neutrons in $\pi$ bonding; (bottom row) on-axis $\sigma$ bonding. The panels in the right-hand column show the corresponding densities. Reproduced from Ref. [21].

\section{Hoyle-state excitations: recent experimental results}

Of the recent experimental results, two approaches aimed at elucidating the first excitation of the Hoyle state point to an $I^{\pi}=2^{+}$resonance between 9.5 and $10 \mathrm{MeV}$.

The first mechanism used is that of inelastic excitation using beams of protons and $\alpha$ particles. The main difficulty is the the nearby broad $I^{\pi}=0^{+}$resonance $(\Gamma=3.0 \mathrm{MeV})$ at $10.3 \mathrm{MeV}$. Measuring at small angles using the K600 high-resolution spectrometer at iThemba LABS, South Africa, has 
enabled the 7-12 MeV excitation region to be measured with precision at the first minimum in the angular distribution of the broad $I^{\pi}=0^{+}$state. The results obtained, summarised in the spectra shown in Fig. 4, conclude that a $I^{\pi}=2^{+}$excitation exists at $9.75 \pm 0.15 \mathrm{MeV}$ with a width of $\Gamma=750 \pm 150$ $\mathrm{keV}$.

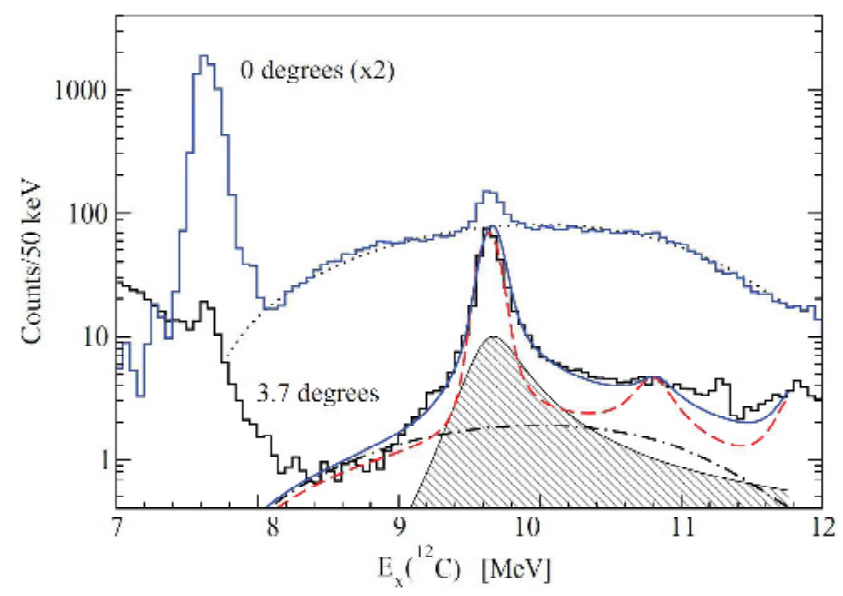

Figure 4. Two measurements are shown corresponding to inelastic $\alpha$-particle scattering at $386 \mathrm{MeV}$ recorded at $0^{\circ}$ (blue stepped line) and $3.7^{\circ}$ (black stepped line). Reproduced from Ref. [22]. Various states in ${ }^{12} \mathrm{C}$ are evident. The $3.7^{\circ}$ data lie in the first minimum of broad $10.3 \mathrm{MeV}, I^{\pi}=0^{+}$ resonance. This state and any other broad components are caracterized with the black dotted curve. The $I^{\pi}=3^{-}$state at 9.641 $\mathrm{MeV}$ is strongly populated in both sets of data. The blue solid line and red-dashed line are $R$-matrix fits to the data with and without the proposed $I^{\pi}=2^{+}$excitation which is represented by the hashed area. The black dot-dashed line is the scaled $I^{\pi}=0^{+}$background used in the (blue line) fit.

In conjunction with the above measurement, since the newly reported state lies close to the known $I^{\pi}=3^{-}$level at $9.641 \mathrm{MeV}$, it is important that the characteristics of this state are also known with high precision. Following a detailed study of the proton inelastic scattering at $66 \mathrm{MeV}$ using the same set-up at iThemba labs, an $R$-matrix fit to the $9.64 \mathrm{MeV}$ region implies a width of $48 \pm 2 \mathrm{keV}$ for the $3^{-}$level [23], considerably larger than the literature value of $34 \pm 5 \mathrm{keV}$ [24].

The second method recently employed to uncover the Hoyle $2^{+}$excitation used ${ }^{12} \mathrm{C}\left(\gamma, \alpha_{0}\right)$ resonance reactions. Gamma-ray energies of 9.1-10.7 MeV were used to observe the angular dependence of various $\gamma$-ray induced $\alpha$-emission cross sections. The results (see Fig. 5) identify a state at $10.03 \pm 0.11 \mathrm{MeV}$ with a width $\Gamma=800 \pm 130 \mathrm{keV}$.

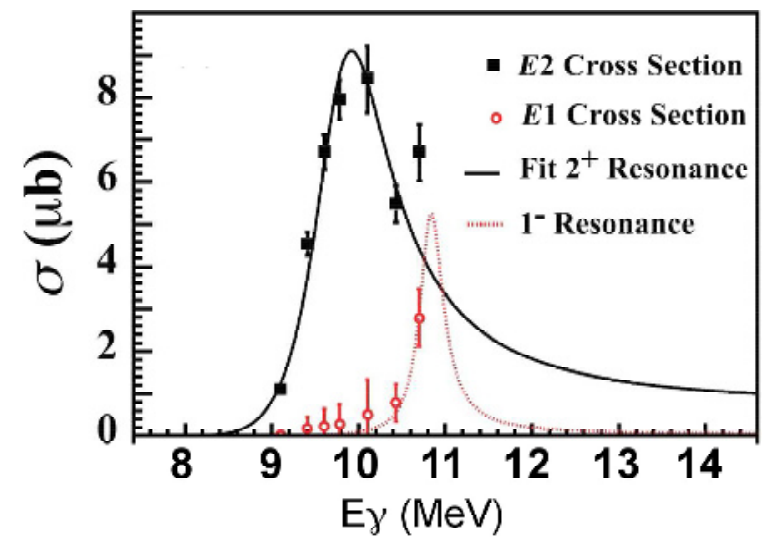

Figure 5. The measured $E 1$ and $E 2$ cross sections. The $I^{\pi}=1^{-}$state at $10.84 \mathrm{MeV}$ $(\Gamma=315 \mathrm{keV})$, shown in red, is also populated in $\gamma$-induced reactions. Reproduced from [25].

These two distinct measurements produce consistent results within approximately one standard deviation. But what about the higher-lying excitations? Recently, searches for such states have pro- 
duced a promising result. Using the two break-up reactions ${ }^{12} \mathrm{C}\left({ }^{4} \mathrm{He},{ }^{4} \mathrm{He}+{ }^{4} \mathrm{He}+{ }^{4} \mathrm{He}\right){ }^{4} \mathrm{He}$ and ${ }^{9} \mathrm{Be}\left({ }^{4} \mathrm{He},{ }^{4} \mathrm{He}+{ }^{4} \mathrm{He}+{ }^{4} \mathrm{He}\right) n$ at ${ }^{4} \mathrm{He}$ beam energies between 22 and $30 \mathrm{MeV}$, excited states in ${ }^{12} \mathrm{C}$ that decay via the ${ }^{8} \mathrm{Be}$ ground-state were reconstructed. In both cases a state at $13.3 \mathrm{MeV}$ was observed with angular distributions indicative of a $I^{\pi}=4^{+}$resonance. A representative spectrum is shown together with the peak shape for the newly observed state in Fig. 6.

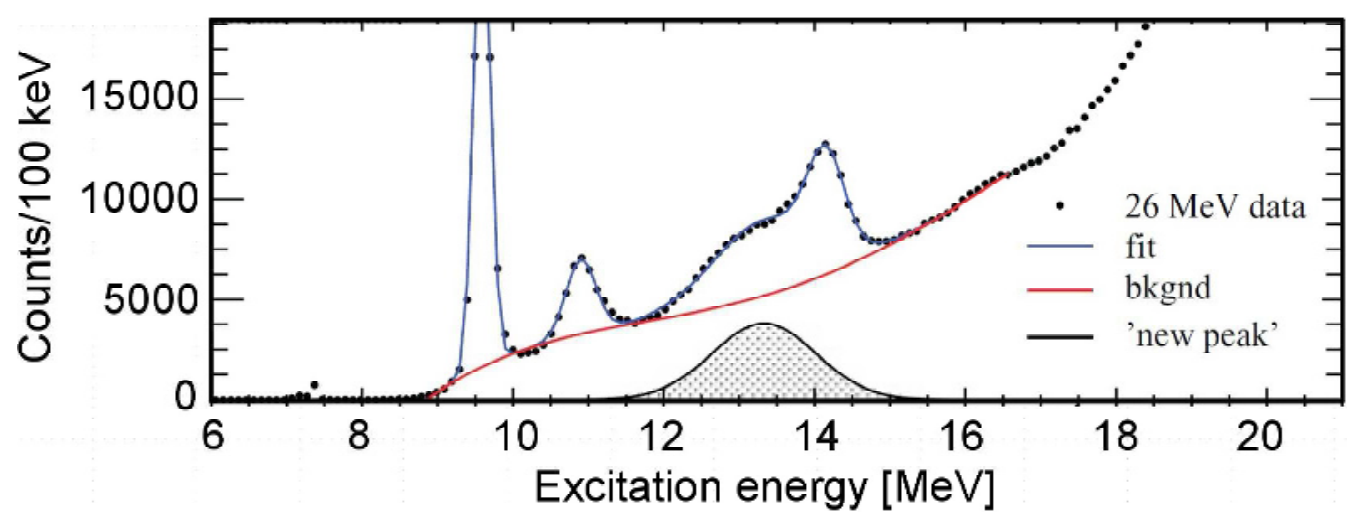

Figure 6. Spectrum from the ${ }^{9} \mathrm{Be}\left({ }^{4} \mathrm{He},{ }^{4} \mathrm{He}+{ }^{4} \mathrm{He}+{ }^{4} \mathrm{He}\right) n$ reaction at $26 \mathrm{MeV}$ (dots). The fit to the data is given by the blue line and includes a 'new' peak at $13.3 \mathrm{MeV}$ as well as the known natural parity states in ${ }^{12} \mathrm{C}$ selected by their decay through the ${ }^{8} \mathrm{Be}$ ground state. The line shape of the $13.3 \mathrm{MeV}$ state is shown by the shaded area. The red solid line corresponds to the polynomial background. Reproduced from [26].

A further experiment performed in the last year at the Birmingham cyclotron, UK, was aimed at firmly establishing the spin of this newly observed $\left(4^{+}\right)$state. Though angular correlations are still under analysis, an interesting feature is the presence of a previously unobserved state in the spectrum at approximately 22-23 MeV. It appears, after an initial analysis, to be even higher spin [27] and is a strong candidate for some higher-lying rotational excitation.

Various predictions for the structure of the Hoyle state have been made, based upon different theoretical frameworks. These include a three- $\alpha$ linear-chain cluster structure calculated by H. Morinaga [28] using the cluster model; a gas-like state of three $\alpha$ particles (i.e. an oblate-shaped configuration with a slightly increased radius compared to the ${ }^{12} \mathrm{C}$ ground-state), see, for example Ref. [29] and a three- $\alpha$ condensate [6], as discussed earlier.

From the recent experimental results summarised here, the separation between the first excitation $\left(2^{+}\right)$and the Hoyle state is approximately $2.1-2.4 \mathrm{MeV}$. Using a charge radius of $1.673 \mathrm{fm}$ for the $\alpha$ particle sub-clusters, the consistency of the Hoyle band with various underlying configurations can be gauged. Two such arrangements are depicted in Fig. 7. The first, a linear chain configuration implies a large moment-of-inertia and, therefore, a small energy gap between the first two band members, $\sim 0.8$ $\mathrm{MeV}$ and is not compatible with the measured energy levels. The second corresponds to a diffuse, oblate arrangement of $\alpha$ particles which has a much lower moment-of-inertia; closer to $2.0 \mathrm{MeV}$. This latter value reproduces the observed excitation energy difference much more closely and suggests that a loose oblate configuration of $\alpha$ particles forms the basic underlying structure of this special state. However, the question remains as to the exact nature of the structure. Is it a gas-like state or a new state of matter formed of condensed $\alpha$ particles? 


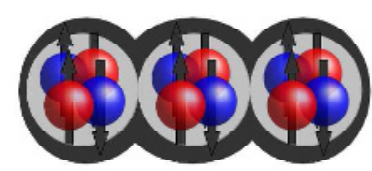

$E(0+)-E(2+)=$

$1 \mathrm{MeV}[0.8 \mathrm{MeV}]$

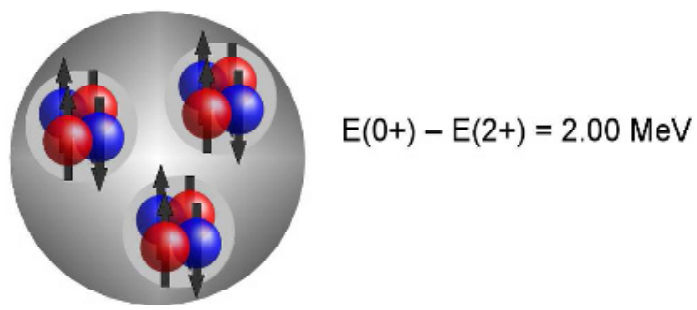

Figure 7. Two possible arrangements for the $\alpha$ particles in the Hoyle band and the corresponding energy separation between the $2^{+}$and $0^{+}$band members. Ref. [30].

\section{Ab initio calculations}

While current experimental data are insufficient to answer this question, models of the nucleus are now sufficiently advanced that with modern computing power, a nucleus containing twelve nucleons can be calculated from a bare nucleon-nucleon potential. Such so-called ab initio approaches are now able to calculate the Hoyle state directly and predict its structure. Using Effective Field Theory (EFT) on the lattice, Epelbaum and co-workers [31] have been able to do just that and the results suggest a bent-arm configuration, reproduced from their paper in Fig. 8. Furthermore, the lattice calculations are able to predict charge radii and electromagnetic transition rates. These quantities enable future experimental results to be benchmarked against the current state-of-the-art theoretical calculations and the Hoyle-state configuration will be laid bare.

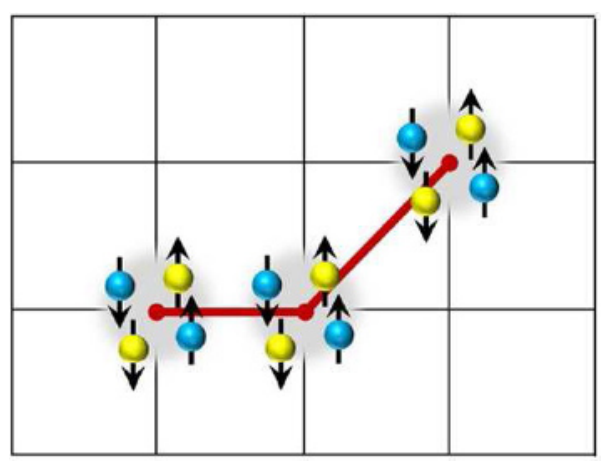

Figure 8. The bent-arm configuration calculated for the Hoyle state by Epelbaum et al. [31].

\section{Summary}

The advent of precision spectroscopy and advances in ab inito theory coupled with increase computing speeds means that the structure of the Hoyle state in ${ }^{12} \mathrm{C}$ is beginning to be uncovered. Recent experimental results have now ruled out some long-standing predictions for its shape (i.e. a linear $\alpha$ chain), distinguishing between an $\alpha$ gas and an $\alpha$-particle condensate require still further precision measurements, e.g. of the Hoyle-state charge radius. The origin of carbon for life excites a curiosity in us that is likely to lead to an answer to the question of the Hoyle-state structure in the very near future. 
INPC 2013

\section{Acknowledgements}

I gratefully acknowledge receipt of a UK, STFC-funded Daphne Jackson Fellowship.

\section{References}

[1] H.O.U. Fynbo et al. Nature 433, 136 (2005).

[2] F. Ajzenberg-Selove, Nucl. Phys. A490, 1 (1988).

[3] F. Hoyle, D. N. F. Dunbar, W. A. Wenzel and W. Whaling, Phys. Rev. 92, 1095 (1953).

[4] R.G. Markham, S.M. Austin and M.A.M. Shahabuddin, Nucl. Phys. A270, 489 (1976).

[5] O.S. Kirsebom et al., Phys. Rev. Lett. 108 (2012) 202501.

[6] A. Tohsaki, H. Horiuchi, P. Schuck, and G. Röpke, 87, 192501 (2001).

[7] W. von Oertzen, Martin Freer and Yoshiko Kanada-En ‘yo, Phys. Rep. 432, 43 (2006).

[8] Martin Freer, Rep. Prog. Phys. 70, 2149 (2007) and references therein.

[9] W. von Oertzen, Eur. Phys. J. A11, 403 (2001).

[10] K. Ikeda, N Tagikawa and H. Horiuchi Prog. Theor. Phys. (Suppl.), extra number, 464 (1968).

[11] R. Nilson, W.K. Jentschke, G.R. Briggs, R.O. Kerman and J.N. Snyder, Phys. Rev. 109, 850 (1958).

[12] T. Teichmann and E.P. Wigner, Phys. Rev. 87, 123 (1952).

[13] R.B. Wiringa, S.C. Pieper, J. Carlson and V.R. Pandharipande, Phys. Rev. 62, 014001 (2000).

[14] Martin Freer, Scholarpedia, 5 (6), 9652 (2010).

[15] C. Wheldon, Tz. Kokalova, W. von Oertzen et al., Eur. Phys. J. A26, 321 (2005).

[16] Y. Funaki et al., Phys. Rev. C67, 051306 (2003).

[17] H. Matsumura and Y. Suzuki, Nucl. Phys. A739, 238 (2004).

[18] Tz. Kokalova, N. Itagaki, W. von Oertzen and C. Wheldon, Phys. Rev. Lett. 96, 192502 (2006).

[19] Y. Funaki et al. Eur. Phys. J. A24, 321 (2005).

[20] T. Yamada and P. Schuck, Eur. Phys. J A26, 185 (2005).

[21] M. Freer (2010), Scholarpedia, 5(6):9652.

[22] M. Freer, M. Itoh et al., Phys. Rev. C86, 034320 (2012).

[23] Tz. Kokalova et al., Phys. Rev. C87, 057307 (2013).

[24] F. Ajzenberg-Selove, Nucl. Phys. A506, 1 (1990).

[25] W.R. Zimmerman et al. Phys. Rev. Lett. 110152502 (2013).

[26] M. Freer et al., Phys. Rev. C83, 034314 (2011).

[27] D. Marín Lambarri, private communication.

[28] H. Morinaga, Phys. Rev. 101, 254 (1956).

[29] H. Horiuchi, Prog. Theor. Phys. 51, 1266 (1974).

[30] M. Freer, private communication.

[31] E. Epelbaum et al., Phys. Rev. Lett 109252501 (2012). 
